\title{
Broca's area in the human brain is involved in the selection of grammatical gender for language production: evidence from event- related functional magnetic resonance imaging
}

\author{
St. Heim ${ }^{\mathrm{a}, *}$, B. Opitz ${ }^{\mathrm{b}}$, A.D. Friederici ${ }^{\mathrm{a}}$ \\ ${ }^{a}$ Max Planck Institute of Cognitive Neuroscience, P.O. Box 500 355, 04303 Leipzig, Germany \\ ${ }^{b}$ Saarland University, Saarbrücken, Germany
}

Received 10 April 2002; received in revised form 25 April 2002; accepted 26 April 2002

\begin{abstract}
The neural correlates of the selection of grammatical gender during overt picture naming were investigated by eventrelated functional magnetic resonance imaging in the left hemisphere. Relative to simply naming a picture, the production of the definite determiner of the picture name (requiring gender selection) resulted exclusively in pronounced activation of a single region in the superior portion of Broca's area. This activation was not present in contrasts reflecting lexical access (naming a picture vs. saying "jaja" to a smiley) or articulation (saying "jaja" vs. rest). Rather, lexical access activated other inferior frontal regions, insula, fusiform and inferior temporal gyrus. Articulation involved insula, Rolandic operculum, motor and premotor cortex and superior temporal gyrus. The results are discussed with respect to data from studies investigating gender processing during language comprehension. (C) 2002 Elsevier Science Ireland Ltd. All rights reserved.
\end{abstract}

Keywords: Broca's area; Language production; Functional magnetic resonance imaging; Syntax; Gender selection; Inferior frontal gyrus; Rolandic operculum; Lexical access

Models of language production argue that in the course of speech production, the speaker has to go from the concept to the spoken word. According to the serial model by Levelt et al. [8], syntactic gender information becomes activated in the course of this process. There is, however, an ongoing debate whether the activated gender information is selected in any case or only if needed in the following processing steps, i.e. the construction of a noun phrase (see Ref. [13] for a review). As the least common denominator one can state that if gender information is selected for further use in an utterance, it must have been activated prior to selection.

In the past years, much effort has been made to discover the neural correlates underlying syntactic processing. Many studies in the domain of language comprehension showed the involvement of Broca's area (Brodmann's area (BA) 44) $[2-4,10,14]$ and its right homologue $[3,10]$. However, there are few studies concerning the functional neuroanatomy of syntax during language production [5,7]. Levelt and colleagues [7] conducted a magnetoencephalographic study on

\footnotetext{
* Corresponding author. Tel.: +49-3-41-99-40-271; fax: +49-341-99-40-113.

E-mail address: heim@cns.mpg.de (St. Heim).
}

picture naming that did not reveal any activation within Broca's area during the time window of lemma access. A recent positron emission tomography study by Indefrey et al. [5] using overt production, however, provided evidence for the involvement of frontal areas (especially the left BA 6 and Broca's area) for the processing of syntactic information at the word, phrase, and sentence level.

In order to identify the brain regions particularly involved in syntactic gender processing during language production, we conducted an event-related functional magnetic resonance imaging (fMRI) experiment using overt production. Twelve healthy right-handed native German speakers (age, 21-30 years; six females) participated in the experiment. No subject had a known history of neurological, major medical, or psychiatric disorder; none were taking medication at the time of measurement. Informed consent was obtained from all participants. The stimuli were 105 black-on-white line drawings of real objects whose German names had masculine $(n=53)$ or feminine $(n=52)$ gender. In a syntactic task (GEN), the subjects produced the definite determiner of the German picture name, thus selecting grammatical gender information. In a naming task (NAME), subjects named the picture, thereby gaining access to semantic and 


A
\begin{tabular}{|l|l|l|l|l|}
\hline & NULL & BASE & NAME & GEN \\
\hline Lexical access & & & & \\
\hline Gender selection & & & & \\
\hline Phon. code retrieval & & & & \\
\hline Articulation & & & & \\
\hline
\end{tabular}

B
\begin{tabular}{|l|l|l|l|}
\hline & BASE - NULL & NAME - BASE & GEN - NAME \\
\hline Lexical access & & & \\
\hline Gender selection & & & \\
\hline Phon. code retrieval & & & \\
\hline Articulation & & & \\
\hline
\end{tabular}

Fig. 1. Sub-processes of language production as realized $(A)$ in the experimental conditions and $(B)$ in the calculated contrasts.

phonological but (according to La Heji [6]) not to syntactic information. The articulatory baseline task (BASE) was to utter "jaja" in response to the presentation of a smiley. Null events (NULL) during which no stimulus was presented were included as a resting baseline. From these tasks, three contrasts could be calculated (GEN-NAME, NAME - BASE, and BASE - NULL), revealing brain activation for gender selection, lexical access, and articulation, respectively (Fig. 1).

Each trial started with a written cue (presented for $4.5 \mathrm{~s}$ ) in the centre of a computer screen, followed by a fixation cross for a variable period $(0,0.5,1$ or $1.5 \mathrm{~s})$. Then, the picture appeared for $800 \mathrm{~ms}$. Thereafter, the screen remained blank until the next trial, resulting in a trial duration of $10 \mathrm{~s}$. Due to scanner noise, no response latencies could be measured. However, to guarantee a high level of performance, there was a 5 min training session before the acquisition of the fMRI data.

Cushions and stereotactic fixation were used to reduce head motion. Anatomical images (T1-weighted MDEFT sequence: data matrix, $256 \times 256$; TR, $1.3 \mathrm{~s}$; TE, $10 \mathrm{~ms}$ ) and functional images (gradient-echo EPI sequence: data matrix, $64 \times 64$; field of view, $19.2 \mathrm{~cm}$; TE, $30 \mathrm{~ms}$; TR, 2 s) were recorded at $3 \mathrm{~T}$. Functional data were acquired from 12 sagittal slices $(3 \mathrm{~mm} / 1 \mathrm{~mm})$ in the lateral part of the left hemisphere and processed using the software package LIPSIA [9]. Pre-processing included correction for motion, temporal offset between the slices, and baseline correction (temporal highpass filter, 1/40 Hz; spatial Gaussian filter, full width at half-maximum $(F W H M)=5.64 \mathrm{~mm})$. No subjects or slices had to be excluded from the analyses due to motion artefacts (mean motion over complete session: $1.19 \mathrm{~mm}$; SD: $0.72 \mathrm{~mm}$ ). Statistical analyses of the individual 2D-data sets were based on the general linear model. The model equation was convolved with a Gaussian kernel $(\mathrm{FWHM}=4 \mathrm{~s})$. For each subject, the three contrasts were calculated, and individual 2D-SPM $\{Z\}$ were realigned on the corresponding $3 \mathrm{D}$ reference data set and normalized into the Talairach stereotactic space [15]. Group averages were calculated using a random effects model.

In the contrast BASE - NULL, there was significant activation in the articulatory network (motor and premotor cortex (MC/PMC), Rolandic operculum (RO), medial frontal gyrus (MFG), inferior frontal sulcus (IFS) and anterior insula (aIns)) and the parietal operculum (PO). The contrast revealing lexical access (NAME - BASE) yielded activation in frontal areas (inferior frontal gyrus (IFG) and IFS, frontal operculum and aIns), in the temporal cortex (medial temporal gyrus (MTG) and fusiform gyrus (FG)), and in the medial occipital gyrus (MOG). Gender selection (GEN NAME) elicited activation in the superior Broca's area/IFS. Significant deactivation was observed in the RO and the MC (Table 1 and Fig. 2; $P<0.001$, uncorrected).

The results are straightforward. In the contrast representing lexical access (NAME - BASE), activation was

Table 1

Mean Z-scores of regions of interest in the left hemisphere as a function of the experimental contrasts ${ }^{\mathrm{a}}$

\begin{tabular}{|c|c|c|c|c|}
\hline \multirow[t]{2}{*}{ Region of interest (BA) } & \multicolumn{3}{|c|}{ Co-ordinates } & \multirow[t]{2}{*}{ Mean } \\
\hline & $x$ & $y$ & $z$ & \\
\hline \multicolumn{5}{|c|}{ Articulation (BASE minus NULL) } \\
\hline alns & -39 & 2 & 6 & 8.9 \\
\hline RO/PMC & -51 & 1 & 6 & 10.3 \\
\hline $\operatorname{PrCS}(6 / 8)$ & -37 & 8 & 35 & -5.2 \\
\hline STG (42) & -55 & -16 & 7 & 10.5 \\
\hline $\mathrm{MC}$ & -42 & -12 & 35 & 12.2 \\
\hline PO & -39 & -32 & 15 & 8.4 \\
\hline
\end{tabular}

Lexical access (NAME minus BASE)

$\begin{array}{lrrrr}\text { IFG (44) } & -44 & 17 & 26 & 12.4 \\ \text { alns } & -32 & 21 & 6 & 9.4 \\ \text { IFG (45) } & -49 & 17 & 5 & 6.5 \\ \text { IFG (45) } & -42 & 26 & 17 & 10.8 \\ \text { IFS/PrCS } & -37 & 6 & 30 & 11.9 \\ \text { FG } & -38 & -44 & -13 & 11.1 \\ \text { ITG } & -47 & -64 & -2 & 11.5\end{array}$

Gender selection (GEN minus NAME)

$\begin{array}{lrrrr}\text { IFG (44/45) } & -42 & 16 & 22 & 3.2 \\ \text { IFG (45) } & -39 & 26 & 8 & -3.8 \\ \text { RO } & -48 & 4 & 7 & -3.4 \\ \text { MC } & -47 & -9 & 40 & -3.4 \\ \text { mIns } & -37 & 1 & 1 & -4.1 \\ \text { STG (21/22) } & -48 & -14 & 0 & -5.2 \\ \text { STG (22) } & -41 & -41 & 17 & -4.7 \\ \text { MTG (21/37) } & -52 & -39 & -4 & -3.8 \\ \text { MTG (37) } & -52 & -60 & 7 & -4.1\end{array}$

${ }^{a}$ Co-ordinates refer to the Talairach space [15]. alns, anterior insula; BA, Brodmann's area; FG, fusiform gyrus; IFG, inferior frontal gyrus; IFS, inferior frontal sulcus; ITG, inferior temporal gyrus; MC, motor cortex; MFG, medial frontal gyrus; mlns, medial insula; MOG, medial occipital gyrus; MTG, medial temporal gyrus; PrCS, precentral sulcus; PMC, premotor cortex; PO, parietal operculum; RO, Rolandic operculum; STG, superior temporal gyrus; STS, superior temporal sulcus. 


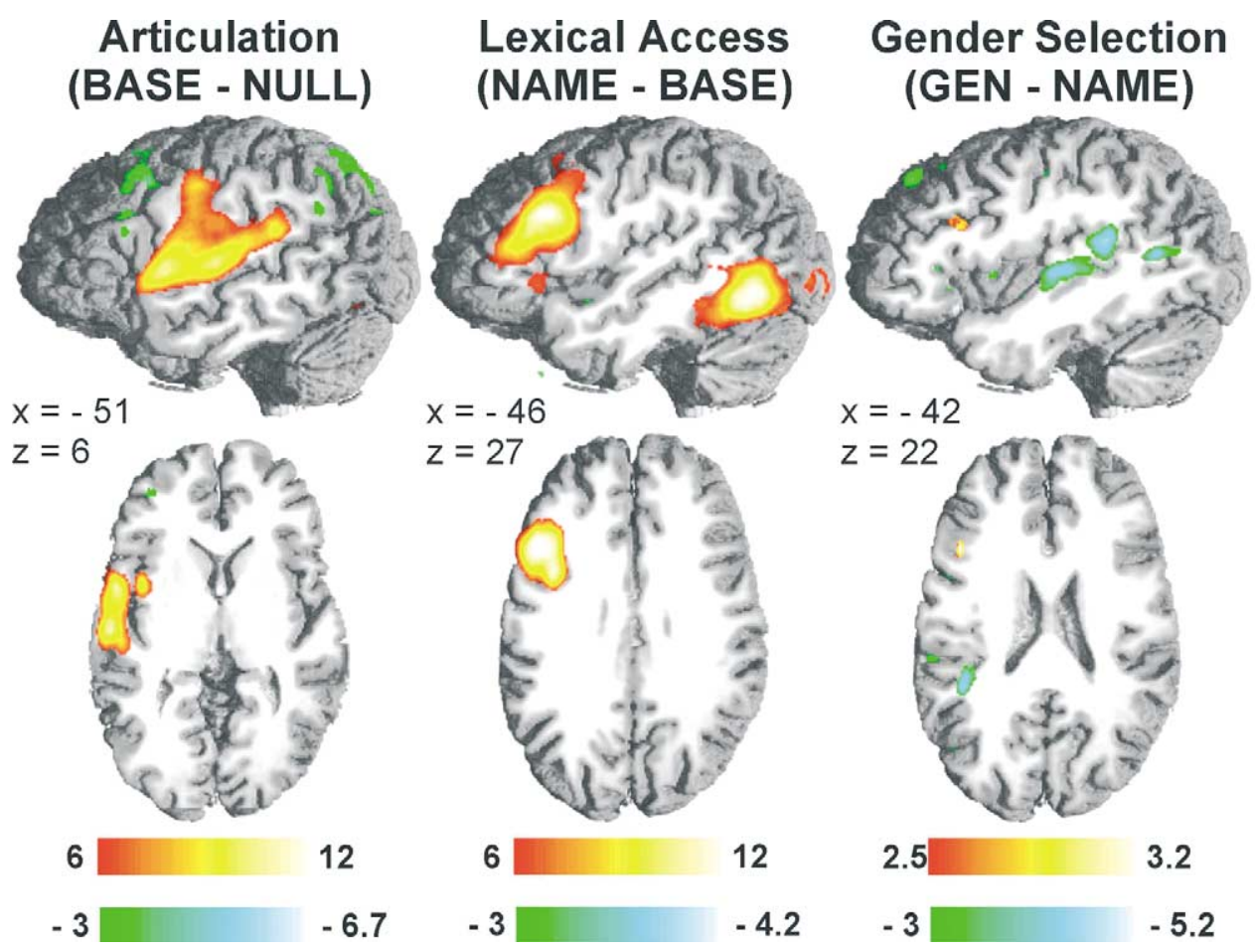

Fig. 2. Statistical parametric maps (SPM $\{Z\}$ ) of the activations in the experimental contrasts, superimposed onto a high-resolution 3D scan of a representative individual brain. The co-ordinates of each particular intersection in the sagittal slices (top row) and the transaxial slices (bottom row) are indicated by the corresponding $x$ - and $z$-values in the Talairach co-ordinate space. The coloured scale bars indicate the activation strength. (Note that, for visualization purposes, different $z$-levels have been chosen for the contrasts.) Left column: Articulation (saying "jaja" minus rest). Middle column: Lexical access (naming a picture minus saying "jaja"). Right column: Gender selection (producing the definite determiner of a picture name minus naming a picture).

observed in prefrontal, temporal, and occipital regions. The foci can be grouped with respect to their function for object identification, semantics, and phonology. Many studies report activation in fusiform and occipital regions in the context of visual object identification [16]. Storage and retrieval of semantic information is closely related to activation in middle temporal and inferior frontal areas (BA 45 and 47) [4]. In contrast, the neural correlates of phonological processing have been localised in the superior portion of Broca's area; in particular, phonological encoding seems to be one of the primary functions of the superior part of BA 44 [18]. In sum, the network activated in the contrast NAME BASE can be considered as representing the different components of lexical access necessary for picture naming.

In the contrast designed to measure articulation (BASE NULL), there is activation in those regions associated with articulatory processes such as MC and PMC and aIns [19]. Interestingly, there was also activation of the $\mathrm{RO}$ in the articulation contrast. This activation was also reported by Indefrey et al. [5] who related it to syntactic processing. However, one could argue that this activation represents articulation or speech planning rather than syntactic processing, for the following reasons. First, this region has been identified to support articulatory and speech planning processes [1]. Second, Indefrey and colleagues [5] found activation in this region in all of their experimental condi- tions, be it the production of a complete sentence (involving syntactic structure building and gender selection), the generation of word lists requiring declension of the adjectives depending on the noun's grammatical gender, or the production of word lists without syntactic information. These three tasks covary not only with the amount of syntactic information needed, but also with the amount of articulation (due to differing numbers of syllables in the required utterance formats: basic forms in the list condition; basic forms plus affixes in the declension condition; declensed forms plus determiners in the sentence condition). Third, in the present study, the same area was also activated in BASE that did not require the processing of any kind of syntactic information, but only articulation. Taken together, the data at hand suggest that activation in the RO could be at least partly due to articulatory effort.

The focus of interest in the present study was on gender selection. This was realized in the contrast GEN - NAME. Again, the results are very clear. While there are deactivations similar to those observed for NAME - BASE (representing lexical access; Table 1), there is one single focus of activation for gender selection which is located in the superior portion of BA $44 / 45$, i.e. the anterior-superior part of Broca's area.

A similar region, however, was reported to support phonological processing in perception as well as in produc- 
tion $[12,18]$. Thus, one might question the interpretation that the activation in the contrast GEN - NAME reflects gender selection. To account for this objection, further analyses were carried out. A possible additional phonological involvement prior to the production of the article in contrast to noun production may have been due to silent production of the complete determiner-noun phrase before uttering the determiner. Such a strategy should result in an increased activation for trials including more syllables. In order to exclude the possibility of the contribution of phonological processes to the activation of the superior Broca's area, we calculated the contrast of disyllabic minus monosyllabic words in the NAME condition, thus detecting the influences of the amount of syllabification on brain activation. Moreover, since the feminine picture names had significantly more syllables than the masculine names (2.1 vs. 1.7; $\left.t_{(104)}=3.68 ; P<0.001\right)$, the contrast masculine minus feminine determiners in the GEN condition was calculated.

The results demonstrate that, in the present experiment, articulatory and phonological effort were correlated with activation in the aIns, the auditory cortex, and the thalamus (and some further regions), but not with activation of the superior portion of Broca's area. Similarly, the production of the determiners of feminine picture names did not require more activation of this area as compared with masculine picture names. Given these results, one can conclude that gender selection but not phonological processes are represented by the activation in the superior portion of Broca's area.

The present data are in line with results from some studies reporting a similar activation for the processing of syntactically complex sentences during comprehension [2,14]; but not with others which investigated the on-line structure building processes either during comprehension $[3,4]$ or during production [5]. How can these different results be accounted for?

The main distinction between these two groups of studies is the level on which syntactic processing is performed. In studies involving syntactic processing on the phrase or sentence level (i.e. extracting word class information [4], syntactic structure building [3,5]), there is activation in the inferior part of Broca's area. In contrast, if processing takes place on the lemma level (as in the present study where gender information is used to simply produce the determiner but not determiner-noun phrase, or identifying the grammatical gender of a word [11]), the superior portion of Broca's area becomes activated. Interestingly, this activation pattern can be found for gender selection in both comprehension [11] and production (the present study), thus providing further evidence for a common neural network for language production and perception in the brain (see Refs. [11,12,17,18] for converging results for the domains of semantic and phonological processing). Thus, these first fMRI results of language production provide some help to integrate the existing and partially contradictory results on syntax processing in the brain and contribute to the attempts to formulate a 'grand unified theory' for the neural correlates underlying language comprehension and production.

[1] Dronkers, N.F., A new brain region for coordinating speech articulation, Nature, 384 (1996) 159-161.

[2] Fiebach, C., Schlesewsky, M. and Friederici, A.D., Syntactic working memory and the establishment of filler-gap dependencies: insights from ERPs and fMRI, J. Psycholinguist. Res., 30 (2001) 321-338.

[3] Friederici, A.F., Meyer, M. and von Cramon, D.Y., Auditory language comprehension: an event-related fMRI study on the processing of syntactic and lexical information, Brain Lang., 74 (2000) 289-300.

[4] Friederici, A.F., Opitz, B. and von Cramon, D.Y., Segregating semantic and syntactic aspects of processing in the human brain: an fMRI investigation of different word types, Cereb. Cortex, 10 (2000) 698-705.

[5] Indefrey, P., Brown, C.M., Hellwig, F., Amunts, K., Herzog, H., Seitz, R.J. and Hagoort, P., A neural correlate of syntactic encoding during speech production, Proc. Natl. Acad. Sci. USA, 98 (2001) 5933-5936.

[6] La Heji, W., Mak, P., Sander, J. and Willeboordse, E., The gender-congruency effect in picture-word tasks, Psychol. Res., 61 (1998) 209-219.

[7] Levelt, W.J.M., Praamstra, P., Meyer, A.S., Helenius, P. and Salmelin, R., A MEG study of picture naming, J. Cogn. Neurosci., 10 (1998) 533-567.

[8] Levelt, W.J.M., Roelofs, A. and Meyer, A.S., A theory of lexical access, Behav. Brain Sci., 22 (1999) 1-75.

[9] Lohmann, G., Müller, K., Bosch, V., Mentzel, H., Hessler, S., Chen, L. and von Cramon, D.Y., LIPSIA - a new software system for the evaluation of functional magnetic resonance images of the human brain, Comput. Med. Imaging Graph., 25 (2001) 449-457.

[10] Meyer, M., Friederici, A.F. and von Cramon, D.Y., Neurocognition of auditory sentence comprehension: event related fMRI reveals sensitivity to syntactic violations of task demand, Cogn. Brain Res., 9 (2000) 19-33.

[11] Miceli, G., Turriziani, P., Caltagirone, C., Capasso, R., Tomaiuolo, F. and Caramazza, A., The neural correlates of grammatical gender: an fMRI investigation, J. Cogn. Neurosci., 14 (2002) 618-628.

[12] Price, C.J., Moore, C.J. and Friston, K.J., Subtractions, conjunctions, and interactions in experimental design of activation studies, Hum. Brain Mapp., 5 (1997) 264-272.

[13] Schriefers, H. and Jescheniak, J.D., Grammatical gender in language production, J. Psycholinguist. Res., 28 (1999) 575-600.

[14] Stromswold, K., Caplan, D., Alpert, N. and Rauch, S., Localization of syntactic comprehension by positron emission tomography, Brain Lang., 52 (1996) 452-473.

[15] Talairach, J. and Tournoux, P., Co-planar Stereotaxic Atlas of the Human Brain, Thieme, Stuttgart, 1988.

[16] van Turennout, M., Ellmore, T. and Martin, A., Long-lasting cortical plasticity in the object naming system, Nat. Neurosci., 3 (2000) 1329-1334.

[17] Vandenberghe, R., Price, C., Wise, R., Josephs, O. and Frackowiak, R.S.J., Functional anatomy of a common semantic system for words and pictures, Nature, 383 (1996) 254-256.

[18] Wise, R.J.S., Greene, J., Büchel, C. and Scott, S., Brain regions involved in articulation, Lancet, 7 (1999) 1057-1061.

[19] Zatorre, R.J., Meyer, E., Gjedde, A. and Evans, A.C., PET studies of phonetic processing in speech: review, replication, and reanalysis, Cereb. Cortex, 6 (1996) 21-30. 